\title{
Structural correlations and melting of B-DNA fibers
}

\author{
Andrew Wildes, ${ }^{1}$ Nikos Theodorakopoulos, ${ }^{2,3}$ Jessica Valle-Orero, ${ }^{1,4}$ Santiago Cuesta-López, ${ }^{4}$ \\ Jean-Luc Garden, ${ }^{5}$ and Michel Peyrard ${ }^{4}$ \\ ${ }^{1}$ Institut Laue Langevin, B.P. 156, 6 rue Jules Horowitz, F-38042 Grenoble Cedex 9, France \\ ${ }^{2}$ Theoretical and Physical Chemistry Institute, National Hellenic Research Foundation, Vasileos Constantinou 48, G-116 35 Athens, Greece \\ ${ }^{3}$ Fachbereich Physik, Universität Konstanz, D-78457 Konstanz, Germany \\ ${ }^{4}$ Université de Lyon, Ecole Normale Supérieure de Lyon, Laboratoire de Physique CNRS UMR 5672, 46 allée d'Italie, \\ F-69364 Lyon Cedex 7, France \\ ${ }^{5}$ Institut Néel, CNRS, Université Joseph Fourier, 25 rue des Martyrs, B.P. 166, F-38042 Grenoble Cedex 9, France
}

(Received 14 March 2011; published 30 June 2011)

\begin{abstract}
Despite numerous attempts, understanding the thermal denaturation of DNA is still a challenge due to the lack of structural data on the transition since standard experimental approaches to DNA melting are made in solution and do not provide spatial information. We report a measurement using neutron scattering from oriented DNA fibers to determine the size of the regions that stay in the double-helix conformation as the melting temperature is approached from below. A Bragg peak from the B form of DNA is observed as a function of temperature and its width and integrated intensity are measured. These results, complemented by a differential calorimetry study of the melting of B-DNA fibers as well as electrophoresis and optical observation data, are analyzed in terms of a one-dimensional mesoscopic model of DNA.
\end{abstract}

DOI: 10.1103/PhysRevE.83.061923

PACS number(s): 87.14.gk, 87.15.Zg, 87.64.Bx

\section{INTRODUCTION}

The x-ray diagrams published by Wilkins et al. [1] and Franklin et al. [2] in the same issue of Nature as the famous paper by Watson and Crick [3] describing the structure of DNA revealed the significance of the fiber form of DNA in providing oriented samples necessary for structural studies. These images, showing the cross pattern typical of a helix and two strong spots associated with the stacking of bases in B-DNA, however, illustrate only one aspect of the molecule, its average static structure. In reality, the DNA molecule is a highly dynamical object. Its base pairs fluctuate widely. The lifetime of a closed base pair is only of the order of $10 \mathrm{~ms}$ [4]. The local opening of the pairs is important for biological function, as it allows reading of the genetic code. When the temperature is raised above the physiological range, thermally induced base-pair openings become more cooperative, leading to so-called "DNA bubbles," that is, open regions which may extend over tens of base pairs. At sufficiently high temperatures they extend over the full molecule and the two strands separate from each other. For a physicist the thermal denaturing of DNA, also known as DNA "melting," is a phase transition, which is particularly interesting because it occurs in an essentially one-dimensional system. DNA melting started to attract attention soon after the discovery of the double-helix structure $[5,6]$ and was widely studied, providing insights into the interactions within DNA, the influence of the base-pair sequence on DNA unwinding, and the effect of the solvent on DNA stability [7]. It has recently attracted renewed interest, thanks to high-resolution melting methods [8] whereby precise denaturing profiles provide a new tool for biology laboratories.

Despite numerous studies, the understanding of this remarkable thermodynamic phase transition is still a theoretical challenge. Statistical physics of DNA thermal denaturing has a long history [7] because it raises the fundamental question of a phase transition in a one-dimensional system and, also, has practical applications such as the design of polymerase chain reaction probes or high-resolution melting studies for biology. Models for DNA denaturation fall into two classes. The first is Ising models, which treat a base pair as a two-state system, which is either closed or open. This is the case for the prototype Poland-Scheraga model [9]. Those models are appealing for their simplicity and because their parameters have been well calibrated. However, their drawback is that they require a large number of phenomenological parameters, and for genomic sequences, the calculation can become heavy due to nonlocal entropy contributions. The second class of models goes beyond a description in terms of two-state systems by incorporating some elements of the structure. The Peyrard-Bishop-Dauxois (PBD) model [10] is still simple because it represents the status of a base pair by a single real number measuring the stretching of the bond between the bases but, nevertheless, contains a minimal structural information relevant for structure factor calculations. In its simplest version this model allows fast calculation of melting curves of long natural DNAs with only seven parameters [11]. However, the success of different models in describing complex melting profiles $[11,12]$ shows that the correct fit of those curves is not a sufficient test to validate a theory. Examination of further observables, with a more direct link to the underlying structural details, appears necessary. As for other classes of phase transitions in physics, such as magnetic systems, an important feature that characterizes the nature of a transition is the growth of the size of the correlated domains as the transition is approached [13].

Traditional methods for investigation of the DNA melting transition cannot provide this kind of spatial information. The standard experimental method is to record the sharp increase in UV absorbance at $260 \mathrm{~nm}$ which is associated with the unstacking of the base pairs, while slowly heating a dilute DNA solution. Other approaches rely on circular dichroism measurements or calorimetric studies that measure the heat absorbed at the transition. Although melting curves, showing the fraction of open base pairs versus temperature, may exhibit a multistep behavior related to the sequence, none of the 
experiments are actually sensitive to spatial information, such as the size of the intact regions of the double helix.

This structural information, which is essential to understanding the nature of a phase transition, has been lacking. Neutron scattering can provide this missing piece of information, provided the experiment can be performed on an oriented DNA sample. As shown by the historical studies that revealed the double-helical structure $[1,2]$, this is possible with fiber diffraction. The methods for producing fiber samples have been refined, by controlling the ionic and water content, so it is now possible to make high-quality fibers with various configurational structures [14,15].

Here we report a measurement using neutron scattering from oriented DNA fibers (Sec. II) to determine the size of the regions that stay in the double-helix conformation as the melting temperature is approached from below and we show how it can be analyzed in terms of the onedimensional mesoscopic PBD model of DNA [10] (Secs. III and IV). We recently published a brief report of these results [16], which are presented here with further data and discussion.

\section{EXPERIMENTS}

Due to the regular stacking of the base pairs, the B form of DNA can be viewed as a one-dimensional diffraction grating. This is reflected in the strong Bragg peak for a longitudinal component of the scattering vector $Q_{\|} \approx 1.87 \AA^{-1}$, associated with the average distance $a=3.36 \AA$ between consecutive base pairs. The principle of our experiment is simple: by following the evolution of this peak as the temperature is raised from room temperature to the denaturation temperature $T_{c}$, we can monitor the breaking of this "diffraction grating" into pieces separated by denatured regions, where the base stacking is destroyed. We expect a strong broadening of the diffraction peak as the $T_{c}$ is approached. The width of the peak allows us to determine how the average size of the intact double-helical domains evolves when DNA approaches its denaturation temperature, which is critical information for a theoretical analysis of the transition. The interesting feature of this method, which focuses on a single, intense, diffraction peak, is that we precisely collect the information of interest in a measurement which is only weakly perturbed by sample imperfections. In fibers, the B form of DNA is semicrystalline [15]. The misalignment of the DNA molecules has been estimated to be less than $5^{\circ}$ [17]. Its effect on the projection of the base-pair distance on the axis is less than $0.5 \%$, that is, negligible with respect to other effects such as variation of the inter-base-pair distance as a function of sequence. As a result, for a cut in reciprocal space along the fiber axis, the width of the Bragg peak is not affected. Moreover, by performing a scan off-center, that is, with a scattering vector $\mathbf{Q}$ which has a nonzero component orthogonal to the molecular axis, we can also probe the displacement of the base pairs in the transverse direction. Such a scan is not immune from the influence of the misalignment of molecules which broaden the peak, but it provides interesting data on fluctuations due to the opening of base pairs in the vicinity of the thermal denaturation.

\section{A. Materials and methods}

\section{Sample preparation}

Samples were made from natural DNA extracted from salmon testes (Fluka). The DNA had been oriented using a "spinning" technique [14], whereby the DNA is precipitated out of a $0.4 M$ lithium salt solution, drawn to a fiber, and then wound around a bobbin to make a film of parallel fibers. The samples are then dried, cut from the bobbin, and stored for a number of weeks in an atmosphere humidified to $75 \%$ using ${ }^{2} \mathrm{H}_{2} \mathrm{O}$. This fixes the water content of the DNA, ensuring a B-form configurational structure, and significantly reduces incoherent neutron scattering from protonated hydrogen in the sample. The B form was confirmed using X-ray and neutron diffraction.

Neutron scattering samples were folded in concertina fashion, thus preserving the fiber axis direction. Samples were then placed in a niobium envelope and sealed between aluminum plates using lead wire for the seal. The sample cassette was therefore airtight, which maintained the water content throughout the experiment. The sample mass was $\sim 1$ g.

\section{Neutron scattering}

Preliminary diffraction measurements to establish the configurational form were carried out using the IN3 threeaxis spectrometer at the Institut Laue-Langevin, France. The instrument was configured with a pyrolytic graphite (PG) monochromator and analyzer, and the wavelength was set to $2.36 \AA$ ( $\equiv 14.7 \mathrm{meV}$ ). The $\mathrm{Q}$ resolution was defined by 60-min collimation before and after the sample, and higher order wavelength contamination was suppressed using a PG filter. The instrument was used to measure reciprocal space maps, and an example is shown in Fig. 1.

The neutron three-axis spectrometer IN8, also at the ILL, was used to measure the main Bragg peak as a function

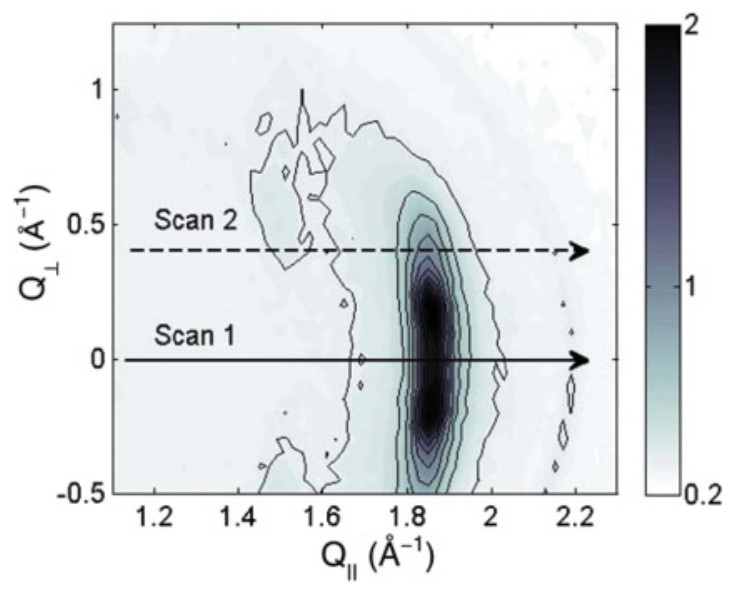

FIG. 1. (Color online) Reciprocal space map of B-form Li-DNA. Axes are the momentum transfer parallel $\left(Q_{\|}\right)$and perpendicular $\left(Q_{\perp}\right)$ to the fiber axis. A strong Bragg peak is observed at $Q_{\|}=2 \pi / a$, where $a \approx 3.36 \AA$ is the average distance between the base pairs along the fiber axis. A powder diffraction peak, coming from the lead wire used to seal the cassette, is just visible at $Q=2.2 \AA$. Also marked are the two standard scans that were repeated at all temperatures. This figure is identical to Fig. 2 top part, in Ref. [16]. 
of temperature. This instrument was configured with a PG monochromator delivering an incident wavelength of $1.53 \AA$ ( $\equiv 35 \mathrm{meV}$ ). The Q resolution was defined with 40-min collimation before and after the sample and was measured by making a reciprocal space map of the (111) Bragg peak from a silicon single crystal. No energy analysis was used, and the static approximation was assumed to hold for the measurements. Temperature control was achieved using a liquid helium cryofurnace.

Two scans were repeated at all temperatures. Their trajectories were calculated for nominally elastic scattering and are shown in Fig. 1. Scan 1 was along the fiber axis, through the center of the Bragg peak. The $Q_{\perp}$ for scan 2 was chosen such that, when $Q_{\|}=2 \pi / a$, the direction of the scattered beam would be perpendicular to the fiber axis. This type of scan has been used to measure critical phase transitions in low-dimensional magnets and assists the static approximation [13]. The temperature steps close to the melting transition were very small $(0.1 \mathrm{~K})$ and measurements at a given temperature were repeated numerous times to ensure thermal equilibrium and reproducibility. Examples of the scans at different temperatures are shown in Fig. 2.

The data were fitted with the Lorentzian function

$$
S\left(Q_{\|}\right)=\int_{-\infty}^{\infty} S\left(Q_{\|}, \omega\right) \mathrm{d} \omega=\frac{I_{0}}{\pi} \frac{\Gamma / 2}{(\Gamma / 2)^{2}+\left(Q_{\|}-Q_{0}\right)^{2}},
$$

where $Q_{0}$ is the peak center, $I_{0}$ is the integrated intensity, and $\Gamma$ is the width. The function was convoluted with the instrument resolution. A second Lorentzian centered at $Q_{\|} \approx 1.5 \AA^{-1}$ was needed to fit the scan 2 data. The amplitudes for these

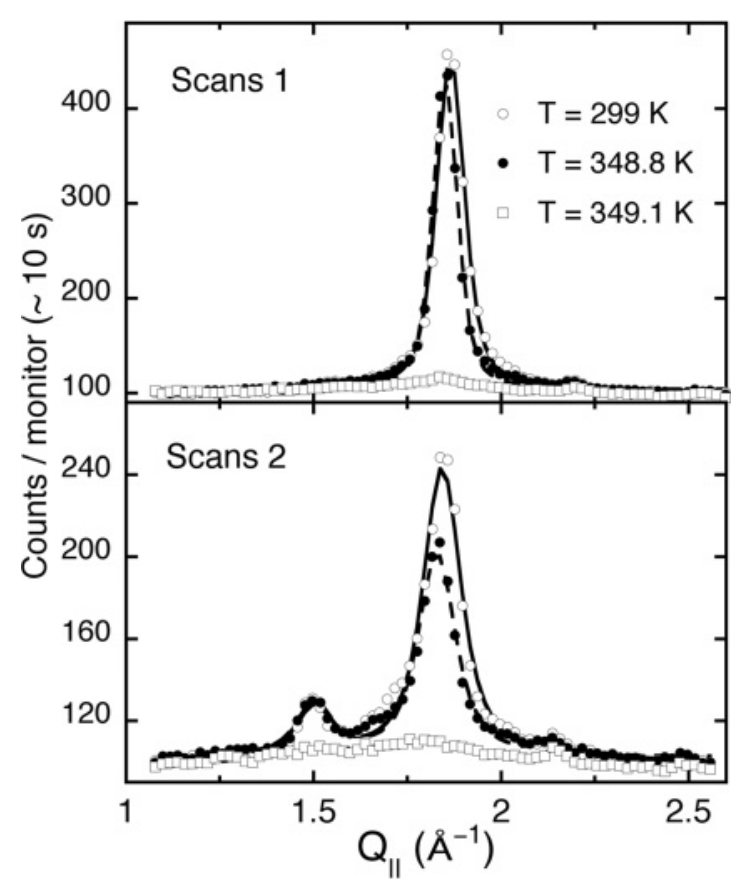

FIG. 2. Examples of the scans shown in Fig. 1. Data at $299 \mathrm{~K}$ represent the starting point for the experiment. The sample is in the melting transition at $348.8 \mathrm{~K}$. The fiber structure has collapsed at 349.1 K. Data were fitted with Eq. (1) and the fits are also shown. This figure is identical to Fig. 2 bottom part, in Ref. [16]. two peaks were free parameters, however, their widths were set to be equal in the fits. Examples of the fits are also shown in Fig. 2.

\section{Calorimetry}

Differential scanning calorimetry (DSC) studies have been performed with a DNA film identical to the one used for neutron scattering, but prepared from a different DNA solution. Two samples were used, with masses of 49 and $45.5 \mathrm{mg}$. The samples cut in the film were rolled and hermetically sealed in the Hastelloy sample tube of a Setaram Micro DSC III calorimeter. The reference tube of this differential calorimeter was empty. After cooling to $278 \mathrm{~K}$ the temperature $T$ was raised to 368 or $383 \mathrm{~K}$ at a rate of $0.6 \mathrm{~K} / \mathrm{min}$, maintained for $10 \mathrm{~min}$ at the maximum temperature, and decreased to $278 \mathrm{~K}$ at the same rate of $0.6 \mathrm{~K} / \mathrm{min}$. The differential heat flux $\Delta \phi$ was measured as a function of time (temperature) and the specific heat was obtained from $\left(\Delta \phi+\tau \frac{d \Delta \phi}{d t}\right) /(d T / d t)$, where $\tau$ is the thermal time constant of the calorimeter (here $\tau=60 \mathrm{~s}$ ) [19].

\section{Optical observations}

A small piece of DNA film was sealed between two glass plates to preserve its water content while it was heated on a hot plate below an optical microscope at the rate of $1 \mathrm{~K} / \mathrm{min}$. The sample was lighted and observed from the top as it went through the denaturation temperature of DNA.

\section{Gel electrophoresis}

A small piece of the sample $(0.01 \mathrm{~g}$ taken before and after heating in the neutron scattering experiment) was dissolved in water. The solution was used to run a standard gel electrophoresis experiment, using a $1 \%$ nondenaturing agarose gel and staining with ethidium bromide. Comparisons with DNA mass ladders were used to measure the length of the DNA fragments in the sample.

A similar experiment was performed with the solution used to prepare the DNA fibers to probe the state of the DNA molecules prior to any treatment.

\section{B. Experimental results}

Figures 2 and 3 illustrate the main result given by neutron diffraction. The integrated intensity of the Bragg peak stays constant from room temperature to about $T=346 \mathrm{~K}$. At this temperature it starts to show a small decrease, occurring in a temperature range of about $3 \mathrm{~K}$, followed by an abrupt drop. A more careful examination of Fig. 3 reveals the following results.

1. The intensity of the peak observed in scan 1 is higher than that of the off-center peak in scan 2, as expected, but its evolution versus temperature is remarkably similar in both scans.

2. The width of the off-center peak (scan 2) is significantly larger than the width at the center of the diffraction spot.

3. In the 300-340 $\mathrm{K}$ temperature range the width of both peaks is essentially constant and even shows a slight decrease, which can be attributed to an annealing of the sample as shown in Fig. 4. In the experiment shown in this figure, another sample was heated up to $340 \mathrm{~K}$, then cooled down to $330 \mathrm{~K}$ 

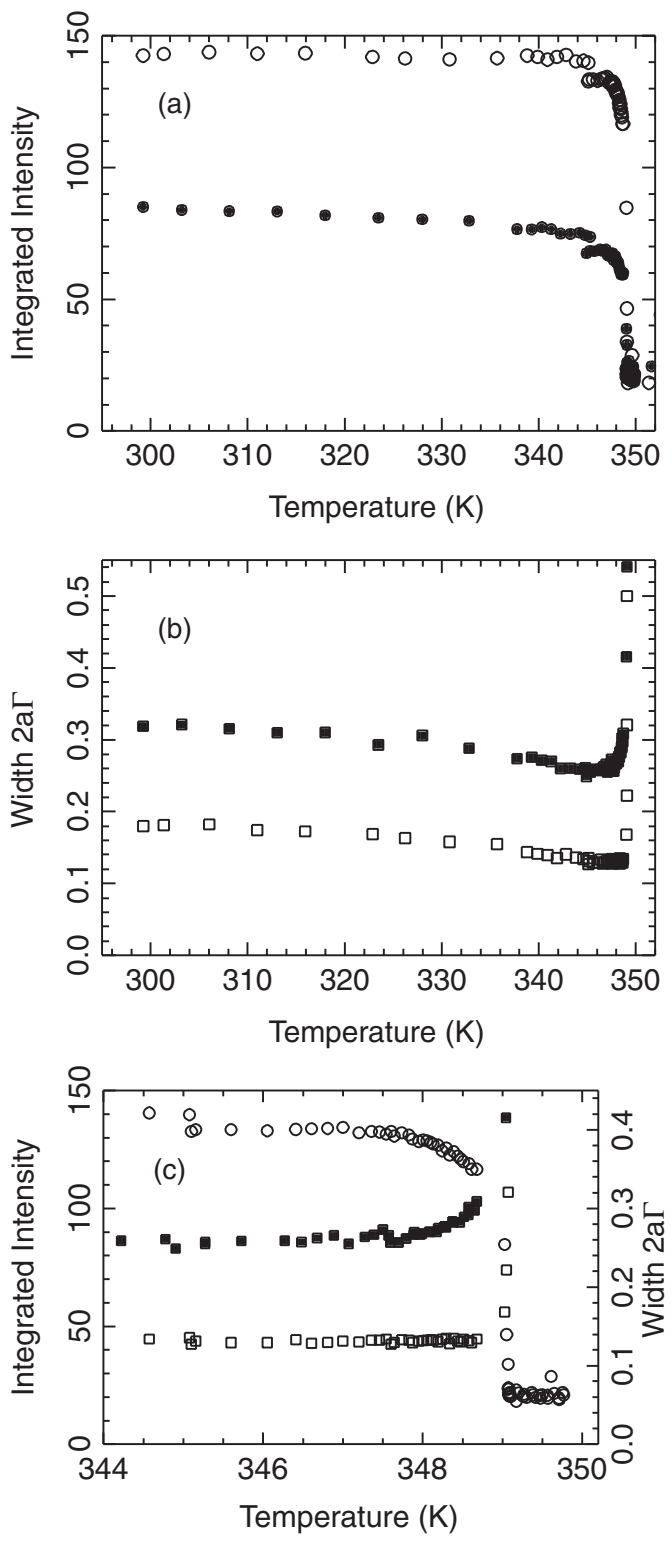

FIG. 3. (a) Integrated intensity and (b) width $2 a \Gamma$ (dimensionless) of the Bragg peaks versus temperature. The small discontinuity at $T \sim 345 \mathrm{~K}$ is due to a small misalignment of the instrument that was discovered and subsequently corrected. Results for scan 1 are shown by open symbols, while results for scan 2 are plotted with filled symbols. (c) Temperature evolution of the intensity (circles) and width of the peak in the immediate vicinity of the melting transition. The evolution of the intensities is the same for both scans, hence data are only shown for scan 1 . Widths are shown for scan 1 (open squares) and scan 2 (filled squares).

and heated up to $340 \mathrm{~K}$ again. During the first heating the width of the peak decreases, but then, in the cooling stage, it keeps its lowest value, as well as during reheating. In this experiment the integrated intensity of the peak stays constant for all temperatures.

4. For scan 1, Fig. 3(c) shows that, in the vicinity of the transition, where the annealing has been completed, the width of the Bragg peak is remarkably constant until the temperature where the intensity drops abruptly. This width does not show any precursor effect, even when the intensity of the mode starts

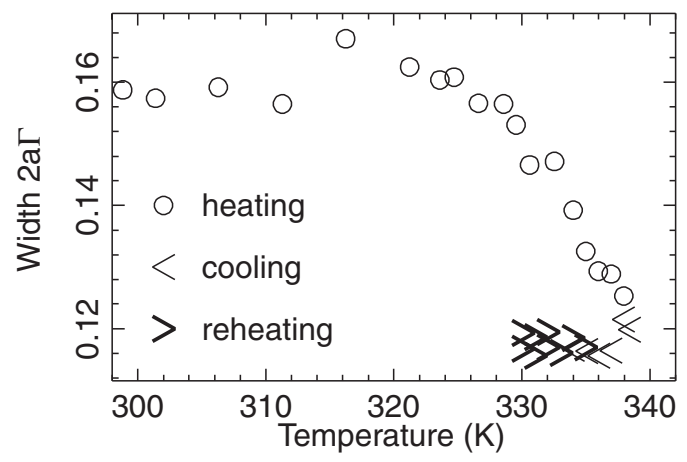

FIG. 4. Experiment showing annealing of the sample due to heating to a moderate temperature. Data correspond to a scan of type 1 . The Bragg peak gets sharper upon heating (open circles) and keeps its lower width if it is subsequently cooled and heated again.

to decrease in the temperature range $346<T<349 \mathrm{~K}$. On the contrary, for scan 2 (off-center) the width of the peak shows a gradual increase in this temperature range, which appears to mirror the decrease in the intensity of the peak.

The drop in the Bragg-peak intensity, shown in Fig. 3, is extremely sharp in terms of temperature but requires some time to complete. This is shown in Fig. 5. When the transition was reached and the intensity started to drop, we stopped cooling and observed the time evolution of the peak at a constant temperature $T=349.05 \pm 0.03 \mathrm{~K}$. Figure 5 shows that the intensity required about $3 \mathrm{~h}$ to stabilize at a low value, and the width was not stabilized before about $6 \mathrm{~h}$.

In the fiber sample the transition that corresponds to the almost-complete vanishing of the Bragg peak associated with
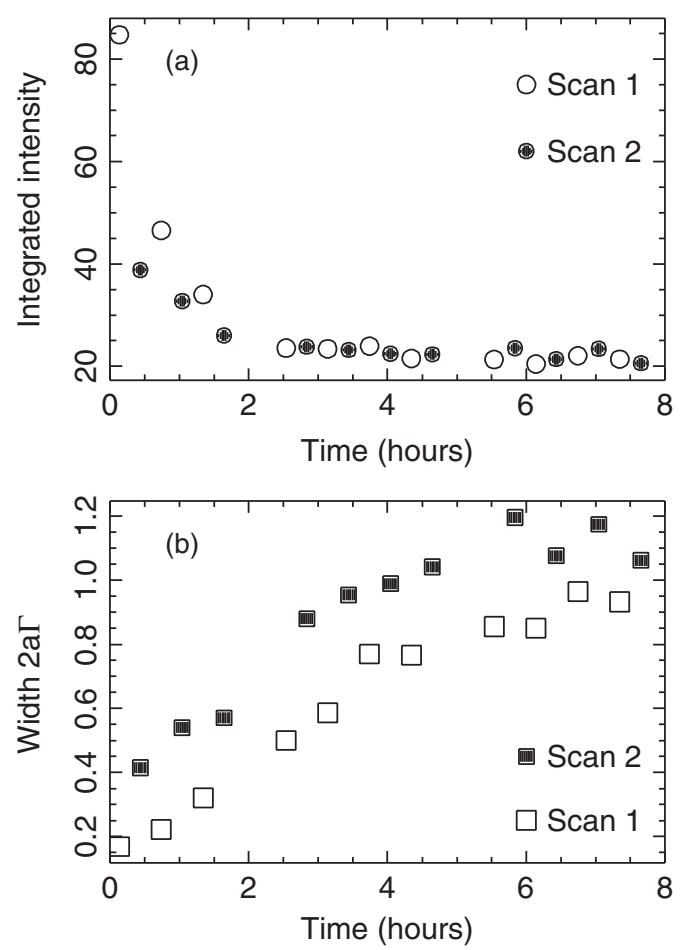

FIG. 5. Time dependence of (a) the integrated intensity (a) and (b) the width of the Bragg peak at a fixed temperature $T=349.05 \pm$ $0.03 \mathrm{~K}$, where the sharp drop in the peak intensity occurs. 
$\left(\times 10^{4}\right)$

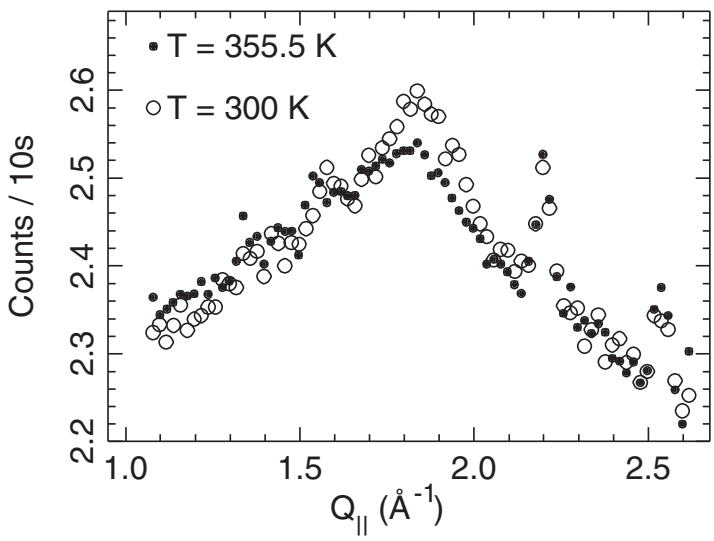

FIG. 6. Plot of the Bragg peak remaining after heating the sample to $355 \mathrm{~K}$ (filled symbols) and after subsequent cooling to room temperature (open symbols). The sharp peak around $2.2 \AA^{-1}$ is due to the aluminium in the sample holder.

the stacking of the base pairs is not reversible. Upon cooling we did not observe a reappearance of the peak, although a very small recovery can be observed in the magnified picture of the Bragg peak recordered at room temperature after cooling as shown in Fig. 6.

Figure 7 shows the specific heat of a DNA film obtained by DSC. The sharp peak at $360 \mathrm{~K}$ can be attributed to the thermal denaturation of DNA, although the transition temperature $T_{c}$ cannot be quantitatively compared with neutron observations because the measurement was done on a different sample. $T_{c}$ strongly depends on external conditions, particularly the ionicity of the solvent [18], so that the shape of the melting curve is more significant than the value of the denaturation temperature when comparing samples. The transition is not reversible, and the sharp peak does not reappear if the sample is cooled and the measurement repeated [20].

The fiber structure of the film is clearly visible in optical microscopy observations of heated DNA films until the denaturation temperature is reached. Then this organized

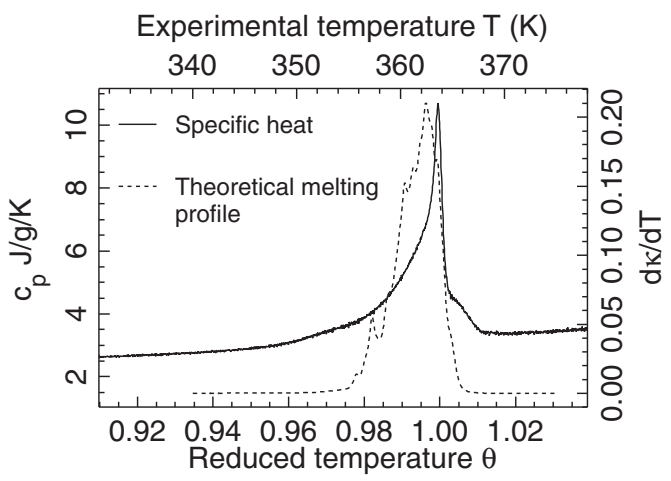

FIG. 7. Specific heat $c_{p}$ of a B-form Li-DNA film similar to the film used in neutron scattering experiments (solid line, left scale) obtained by DSC. The dashed line shows the theoretical denaturation profile of the sequence used for analysis (Sec. III): derivative with respect to temperature of fraction $\kappa$ of open base pairs. This figure is identical to Fig. 1 in Ref. [16]. (a)

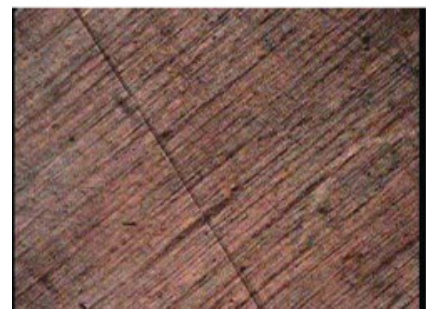

(b)

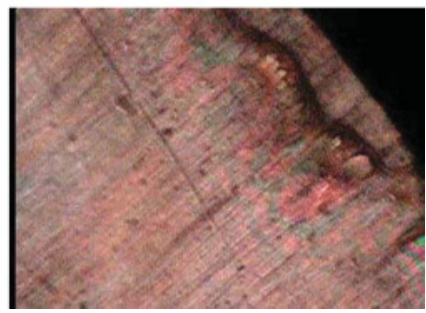

FIG. 8. (Color online) Optical microscopy observation of a piece of film (a) before and (b) after heating above the denaturing temperature of DNA.

structure of parallel fibers is essentially lost and the film tends to shrink (Fig. 8).

Gel electrophoresis shows that the length of DNA molecules in the solution used to prepare the film, or in a piece of film which was not heated, is of the order of $20 \mathrm{~kb}$ or larger. However, the same measurements performed on a piece of film which was heated up to DNA denaturation and film collapse, and subsequently cooled to room temperature, only detects DNA fragments of a few hundreds of bases, indicating that the original molecules have been chopped in the thermal cycle (Fig. 9).

\section{ANALYSIS}

To analyze the neutron diffraction results, we must proceed in two steps. First, we must determine the diffraction pattern of a finite segment of double-stranded DNA, taking into account

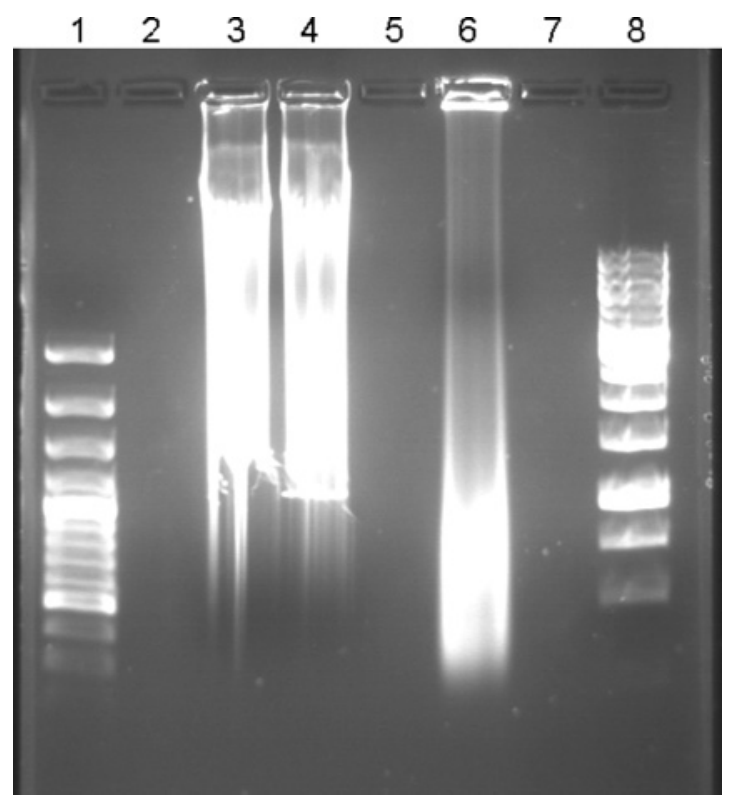

FIG. 9. Electrophoresis image showing the length of DNA molecules before and after heating of the film. From left to right: DNA mass ladder SM0321 (100-3000 base pairs; lane 1); solution used to prepare the DNA film (lane 3); solution prepared from a piece of film that was not heated (lane 4); solution prepared from a piece of film after heating (lane 6); DNA mass ladder SM0311 (250-10 000 base pairs; lane 8 ). Lanes 2, 5, and 7 were not used. 
the local inhomogeneities in its structure which are associated with the base-pair sequence, and the thermal fluctuations. Second, we must study the statistical physics of DNA to determine the size distribution of the closed segments of DNA as a function of temperature.

\section{A. Structure factor of a closed DNA segment}

We consider a structurally disordered linear chain of $M$ sites. Let $a$, the average base-pair spacing, be the average distance between successive sites and $\lambda_{j}$ the local structural deviation from that value between the $j$ th and the $(j+1)$ th sites; the equilibrium positions of the $m$ th and $n$th sites differ by $(m-n) a+\sum_{j=n+1}^{m} \lambda_{m}$. Structural disorder in the transverse direction is similarly expressed by $\eta_{j}$, the local deviation (from $0)$ of the distance between the $j$ th and the $(j+1)$ th sites in the transverse direction. The structure factor of such a finite chain segment is given by

$$
\begin{aligned}
S_{M}(\mathbf{Q})= & \frac{1}{M} \sum_{m, n=1}^{M} e^{i Q_{\|} a(m-n)}\left\langle e^{i \sum_{j=m+1}^{n}\left(Q_{\|} \lambda_{j}+Q_{\perp} \eta_{j}\right)}\right\rangle \\
& \times\left\langle e^{i Q_{\|}\left(x_{m}-x_{n}\right)+i Q_{\perp}\left(y_{m}-y_{n}\right)}\right\rangle,
\end{aligned}
$$

where $x_{m}$ and $y_{m}$ represent, respectively, the longitudinal and transverse displacements of the $m$ th site from its position at thermal equilibrium, and $\mathbf{Q}$ is the scattering vector, having the component $Q_{\|}$along the helix axis and $Q_{\perp}$ orthogonal to it. Equation (2) is a slight generalization of the finite paracrystal theory [21] to account for disorder and motion in the transverse direction. The angle braces denote averages which can be decoupled because the first refers to structural disorder and the second to thermal motion. To a first approximation, structural disorder is modeled by Gaussian variables $\left\{\lambda_{j}\right\}$ and $\left\{\eta_{j}\right\}$ with zero average and

$$
\begin{gathered}
\left\langle\lambda_{i} \lambda_{j}\right\rangle=\left\langle\lambda^{2}\right\rangle \delta_{i j}, \\
\left\langle\eta_{i} \eta_{j}\right\rangle=\left\langle\eta^{2}\right\rangle \delta_{i j}, \\
\left\langle\eta_{i} \lambda_{j}\right\rangle=\chi\left[\left\langle\eta^{2}\right\rangle\left\langle\lambda^{2}\right\rangle\right]^{1 / 2} \delta_{i j},
\end{gathered}
$$

where $\delta_{i j}$ is the Kronecker symbol $\left(\delta_{i j}=1\right.$ if $i=j$ and 0 otherwise). We use estimates of the variances $\left\langle\lambda^{2}\right\rangle$ and $\left\langle\eta^{2}\right\rangle$ obtained from conformational analysis [22] and present alternative calculations for uncorrelated $(\chi=0)$ and correlated $(\chi \neq 0)$ structural disorder. Thermal fluctuations in the longitudinal displacements can be calculated in the harmonic approximation; thermal fluctuations in the transverse direction can also be calculated (cf. the next subsection for a particular model). We describe them in an approximate fashion which takes into account thermal effects due to sequence heterogeneity, that is, local variations in $\xi_{j}=\left\langle y_{j+1}\right\rangle-\left\langle y_{j}\right\rangle$; then

$$
\left\langle e^{i Q_{\perp}\left(y_{m}-y_{n}\right)}\right\rangle \approx e^{-Q_{\perp}^{2}\left\langle\xi^{2}\right\rangle|m-n| / 2},
$$

where $\left\langle\xi^{2}\right\rangle$ is the variance of the $\left\{\xi_{j}\right\}$ values.

Putting the various terms together and performing one of the two summations [21] results in

$$
S_{M}(\mathbf{Q})=M+2 \sum_{n=1}^{M-1}(M-n) \cos \left(Q_{\|} n a\right) e^{-n \Delta},
$$

where

$$
\begin{aligned}
2 \Delta= & Q_{\|}^{2}\left(\left\langle\lambda^{2}\right\rangle+\left\langle\sigma^{2}\right\rangle\right)+Q_{\perp}^{2}\left(\left\langle\eta^{2}\right\rangle+\left\langle\xi^{2}\right\rangle\right) \\
& +2 Q_{\|} Q_{\perp} \chi\left(\left\langle\eta^{2}\right\rangle\left\langle\lambda^{2}\right\rangle\right)^{1 / 2}
\end{aligned}
$$

and $\sigma^{2}=k_{B} T / \mu c_{0}^{2} a^{2}$, the Debye-Waller correction due to longitudinal thermal motion at any temperature $T$ can be obtained from the total DNA mass per base pair $\mu=618$ a.m.u. and the measured [23] sound velocity $c_{0}=2830 \mathrm{~m} \mathrm{~s}^{-1} ; k_{B}$ is the Boltzmann constant.

Near the first Bragg peak, which is where the present experiment focuses, and for sufficiently large cluster sizes, the sum, Eq. (5), can be approximated by

$$
S_{M}^{*}(\mathbf{Q}) \sim M \mathcal{S}^{\prime}(\mathbf{Q}) \doteq M \frac{\sinh \Delta}{\cosh \Delta-\cos \left(Q_{\|} a\right)}
$$

\section{B. Statistical physics of the closed regions of DNA}

To calculate the size distribution of the closed segments of DNA by a statistical physics analysis, we selected the PBD model [10], which is sufficiently simple to allow the analysis of DNA segments of thousands of base pairs but, nevertheless, includes some data on the structure of the molecule which are necessary to calculate the structure factor. Moreover, as it describes the molecule in terms of a Hamiltonian, its parameters are directly linked to physical quantities. Therefore they are easier to determine than for Ising models, although they still need to be refined by comparison with a variety of experimental melting profiles.

The configuration energy of a DNA molecule of $N$ base pairs is written as

$$
H_{y}=\sum_{j=1}^{N-1} W\left(y_{j}, y_{j+1}\right)+\sum_{j=1}^{N} V_{j}\left(y_{j}\right),
$$

where $y_{j}$ represents the stretching of the $j$ th base pair, due to the transverse displacements of the bases. The stacking interaction between adjacent bases is described by the anharmonic potential [10],

$$
W\left(y_{j}, y_{j+1}\right)=\frac{1}{2} k\left[1+\rho e^{-b\left(y_{j}+y_{j+1}\right)}\right]\left(y_{j}-y_{j+1}\right)^{2},
$$

which takes into account the weakening of the interactions when the pairs are broken. The potential $V_{j}\left(y_{j}\right)=D_{j}[1-$ $\left.\exp \left(-\alpha_{j} y_{j}\right)\right]$ is a Morse potential which describes the combined effects of hydrogen-bonding, electrostatic interactions between the charged phosphate groups, and solvent effects on the the $j$ th base pair. The four possible bases, A, T, $\mathrm{G}$, and $\mathrm{C}$, form two types of pairs, A-T, linked by two hydrogen bonds, and $\mathrm{G}-\mathrm{C}$, linked by three hydrogen bonds. Both the stacking interactions $W$ and the intrapair potential $V$ are affected by the sequence of bases. However, although subtle sequence effects on short DNA fragments may require the introduction of different stacking potentials for different interacting pairs [24], the melting curves of long DNA molecules, with several thousands base pairs, can be accurately reproduced by introducing the effect of the sequence in the intrapair potential $V_{j}$ only [11], which drastically reduces the number of model parameters. Therefore in our calculations the stacking interaction is treated as being homogeneous. 
To determine the melting curve or calculate the size of the closed regions, we need to give a quantitative definition of a closed base pair. This can be done by choosing a reference stretching $y_{c}$. Base pair $j$ is considered to be closed if $y_{j}<y_{c}$. We select $y_{c}=1.5 \AA$, which corresponds to a base pair whose stretching is well on the plateau of the Morse potential. The results are weakly dependent on the value of $y_{c}$ provided it is larger than $1 \AA$ because molecular dynamics simulations show that, once a base pair as been stretched to a value that brings it onto the plateau of the Morse potential, it is likely to open widely.

The statistical weight of a given configuration of the molecule thermalized at temperature $T$ is

$$
\begin{aligned}
\mathcal{Z}= & \int_{y_{l_{1}}}^{y_{m_{1}}} d y_{1} \int_{y_{l_{2}}}^{y_{m_{2}}} d y_{2} \ldots \int_{y_{l_{N}}}^{y_{m_{N}}} d y_{N} \\
& e^{-H_{y}\left(y_{1}, y_{2}, \ldots, y_{N}\right) /\left(k_{B} T\right)} .
\end{aligned}
$$

The limits of the lower and upper bounds of the integrals $y_{l_{j}}$ and $y_{m_{j}}$ depend on the particular configuration. Setting $y_{l_{j}}=-\infty$ and $y_{m_{j}}=+\infty$ for all $j$ allows the molecule to explore its full configurational space and $\mathcal{Z}$ is then the partition function $Z$. Setting $y_{l_{j}}=-\infty$ and $y_{m_{j}}=y_{c}$ defines a configuration in which base pair $j$ is closed while $y_{l_{j}}=y_{c}$ and $y_{m_{j}}=+\infty$ define a configuration where it is open. The integrals associated with all those configurations can be easily calculated because the model is one-dimensional and restricted to nearest-neighbor coupling so that, instead of a highly multidimensional integral, one has to compute a chain of one-dimensional integrals $[11,27,28]$ involving a kernel which depends on the stretching at two adjacent sites. Moreover, the speed of the calculation can be significantly increased by expanding the site-dependent kernel on the basis of the eigenfunctions of a reference kernel, for instance, the kernel associated with an A-T base pair [11].

These calculations allow us to obtain the probability $\mathcal{P}(m, i)$ that $m$ adjacent sites, starting at site $i$, are closed,

$$
\begin{aligned}
\mathcal{P}(m, i)= & \frac{1}{Z} \mathcal{Z}\left(y_{1}, y_{2}, \ldots, y_{i-1}, y_{i}<y_{c}, y_{i+1}\right. \\
& \left.<y_{c}, \ldots, y_{i+m-1}<y_{c}, y_{i+m}, \ldots\right),
\end{aligned}
$$

by computing the statistical weight of configurations where restrictions on the integration range are imposed for all sites belonging to that closed region and no restrictions are imposed elsewhere. Then these quantities give the probability $P(m, i)$ that a closed cluster of size $m$, with open ends, exists at site $i$ through

$$
\begin{aligned}
P(m, i)= & \mathcal{P}(m, i)-\mathcal{P}(m+1, i-1) \\
& -\mathcal{P}(m+1, i)+\mathcal{P}(m+2, i-1),
\end{aligned}
$$

from which the probability of having a closed cluster of size $m$ in a DNA segment of $N_{0}$ base pairs is simply

$$
P(m)=\frac{1}{N_{0}} \sum_{i=1}^{N_{0}} P(m, i) .
$$

The average size of a cluster of closed base pairs is then

$$
l_{c}=\frac{\sum m P(m)}{\sum P(m)}=\frac{h}{1-h-P_{0}},
$$

where $h$ is the helix fraction and $P_{0}$ the statistical weight of two consecutive bases being in the closed state. Similar calculations for the open regions of the DNA molecule give the average size of the denatured regions, $l_{o}=(1-h) /(1-$ $\left.h-P_{0}\right)$. These quantities are computed for a range of cluster sizes from 1 to a maximum value $M$. To avoid end effects we study a DNA segment of size $N=N_{0}+2 M$ and the sites $i$ are chosen so that the clusters that we consider are formed of base pairs which are at least $M$ sites away from the ends.

For a natural DNA sample, which may contain millions of base pairs, the intensity observed in a neutron scattering experiment is proportional to

$$
S(\mathbf{Q})=\sum_{m=1}^{\infty} P(m) S_{m}(\mathbf{Q}),
$$

where $S_{m}(\mathbf{Q})$ is given by Eq. (5), and the size of the DNA molecule has been extrapolated to infinity.

In practice, the calculation is performed with segments of natural DNA which include 280000 base pairs, and $P(m, i)$ is computed up to cluster sizes $M$ of a few hundreds of base pairs (typically $M=150)$. It is, however, easy to determine $P(m)$ for large $m$ because it scales exponentially with $m$ [29], as shown in Fig. 10(b). Fitting the numerical data on $\log [P(m)]$ for $40 \leqslant m \leqslant M$ by a straight line, we get $P(m)=P_{0} \zeta^{m}$ for $m>M$, so that, in practice, the calculation of $S(\mathbf{Q})$ by Eq. (15) is expressed as

$$
\begin{aligned}
S(\mathbf{Q})= & \sum_{m=1}^{M} P(m) S_{m}(\mathbf{Q})+\sum_{m=M}^{M_{0}} P_{0} \zeta^{m} S_{m}(\mathbf{Q}) \\
& +\mathcal{S}^{\prime}(\mathbf{Q}) P_{0} \zeta^{M_{0}+1}\left[\frac{M_{0}}{1-\zeta}+\frac{1}{(1-\zeta)^{2}}\right],
\end{aligned}
$$

where the summation for $m>M_{0}\left(M_{0}=1000\right)$ has been calculated analytically using the property that, for large $m$, $S_{m}(\mathbf{Q})$ can be approximated by the limiting form, Eq. (7). The same method can be used to compute the average cluster size $l_{c}$ [Eq. (14)] versus temperature. A typical result is shown in Fig. 10(c). Then the structure factor is fitted with the same Lorentzian expression as the one used to analyze experimental data to determine the integrated intensity $I_{0}$ and width $\Gamma$ of the diffraction peak.

\section{Model parameters}

To analyze the neutron scattering experiments, in principle, we would need to know the base-pair sequence in the sample. As the experiment requires a significant amount of DNA, it can only be performed with natural DNA. The salmon testes DNA that we use is provided without its sequence [30], and even its GC content is only approximately known. It is estimated to be $41.2 \%$.

The theoretical analysis has been tested on different DNA sequences from the genome of Danio rerio (zebrafish) [31] and Pyrococcus abyssi [32]. The results presented in Fig. 10 were obtained with a sequence of 280000 bases, part of the full genome of Pyrococcus abyssi, chosen because its denaturation curve is the closest to the denaturation profile of our samples measured by DSC (Fig. 7). The GC content of this fragment is $44.08 \%$. 
(a)

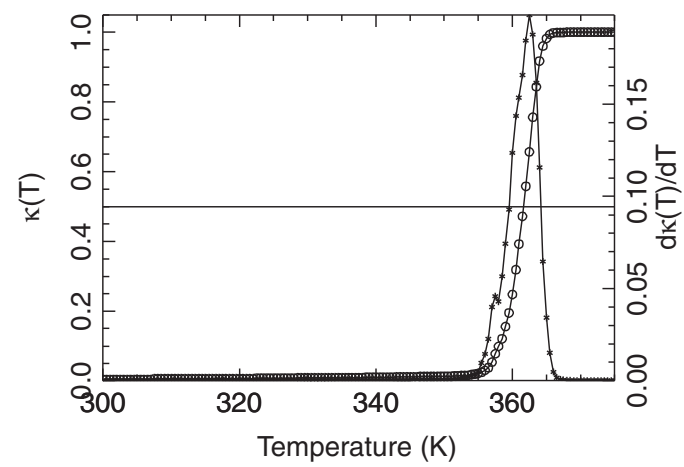

(c)

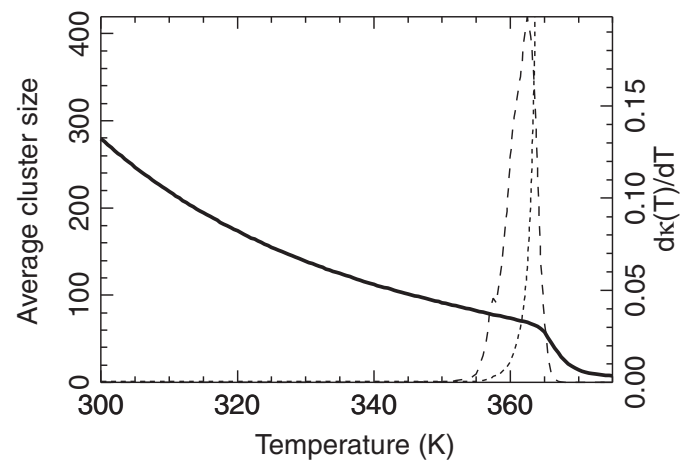

(b)

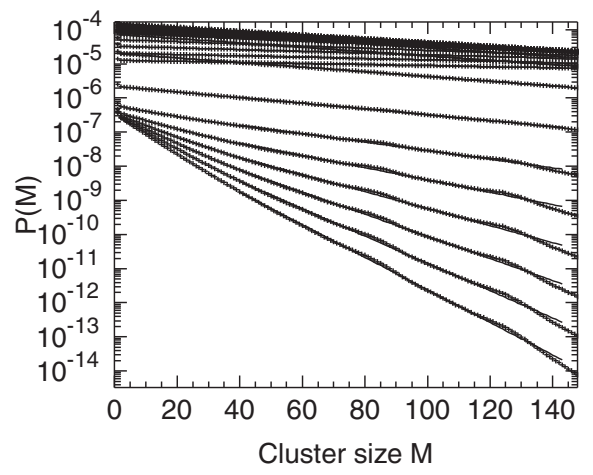

(d)

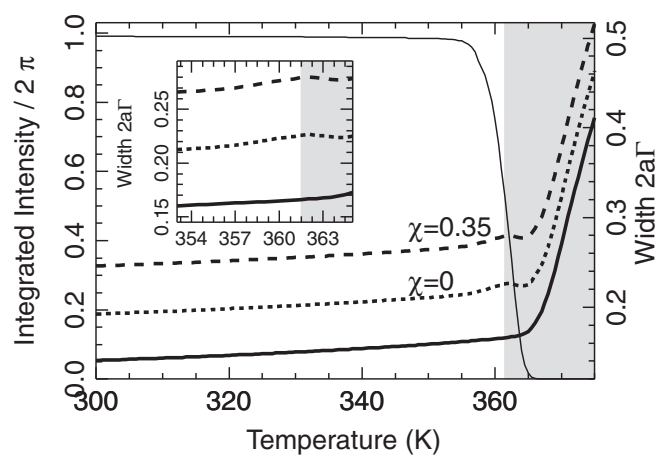

FIG. 10. Theoretical results. (a) Melting curve of a reference DNA segment of 280000 base pairs, part of the genome of Pyrococcus abyssi: circles show the open fraction versus temperature, and stars correspond to its derivative to get the melting profile. (b) Probabilities $P(m)$ versus $m$ at different temperatures in the range $T=330 \mathrm{~K}$ to $T=375 \mathrm{~K}$, on a logarithmic scale. Points are values calculated from the statistical mechanics of the DNA sample, and lines show a linear fit for $40 \leqslant m \leqslant 150$. Curves are plotted every $10 \mathrm{~K}$ from 300 to $340 \mathrm{~K}$ and every $1.5 \mathrm{~K}$ above that range. (c) Average size of closed clusters $l_{c}$ (solid line) and average size of open regions $l_{o}$ (dotted line) versus $T$. The dashed line shows the melting profile on the same temperature scale. (d) Integrated intensity (thin solid line) and width (thick solid line for scan 1, dotted and dashed lines for scan 2) versus temperature. For scan 2, two cases are shown: $\chi=0$, i.e., ignoring the correlation between longitudinal $\lambda_{j}$ and transverse $\eta_{j}$ components of the structural disorder due to the sequence (dotted line); and $\chi=0.35$ (dashed line), which assumes a partial correlation. The shaded area shows the temperature range in which the experimental observations are hindered by melting of the sample film (Sec. IV). Inset: A magnification of the variation of the widths versus temperature in the immediate vicinity of the transition.

Model parameters were obtained from an extensive study of DNA denaturation in various sequences [11], which determined a set of parameters allowing the prediction of melting curves of various DNA sequences to a good accuracy. Experiments to record DNA denaturation curves are generally performed in solution with sodium salt, the $\mathrm{Na}^{+}$ions being necessary to stabilize DNA by screening the repulsions between the charged phosphate groups. However, our neutron scattering experiments were performed with Li-DNA because its secondary structure is more stable, and stays in the B form over a much higher humidity range, than that of Na-DNA $[25,26]$. Therefore the parameters of the AT and GC Morse potentials cannot be determined unambiguously. The values that we use correspond to a high sodium content. They were chosen on the basis of the shape of the melting curve rather than the value of the denaturation temperature $T_{c}$. This is why comparison between theory and experiments must be made with the reduced temperature $\theta=T / T_{c}$ rather than with the actual temperature. The parameters selected for the analysis are $k=0.00045 \mathrm{eV} / \AA^{2}, \rho=50, b=0.20 \AA^{-1}, \alpha_{\mathrm{AT}}=$
$4.2 \AA^{-1}, \quad \alpha_{\mathrm{GC}}=6.9 \AA^{-1}, \quad D_{\mathrm{AT}}=0.12905 \mathrm{eV}$, and $D_{\mathrm{GC}}=$ $0.16805 \mathrm{eV}$.

\section{Results}

Figure 10 summarizes the main results of the theoretical analysis by showing the variation versus temperature of the integrated intensities and widths of the Bragg peaks in scans 1 and 2, as predicted by the model. As expected, the intensity shows a sharp drop near the denaturation transition. It reflects the openings of the base pairs that break the clusters of stacked pairs, giving rise to the investigated Bragg peak, and therefore reduces the number of scattering sites. As a result, the integrated intensity of the peak almost provides a quantitative measure of the helix fraction of DNA because, as shown by Eq. (7), for sufficiently large clusters, the structure factor is proportional to the number of sites in a cluster. Moreover Fig. 10(c) shows that the size of the clusters drops significantly only in the last stage of denaturation. 


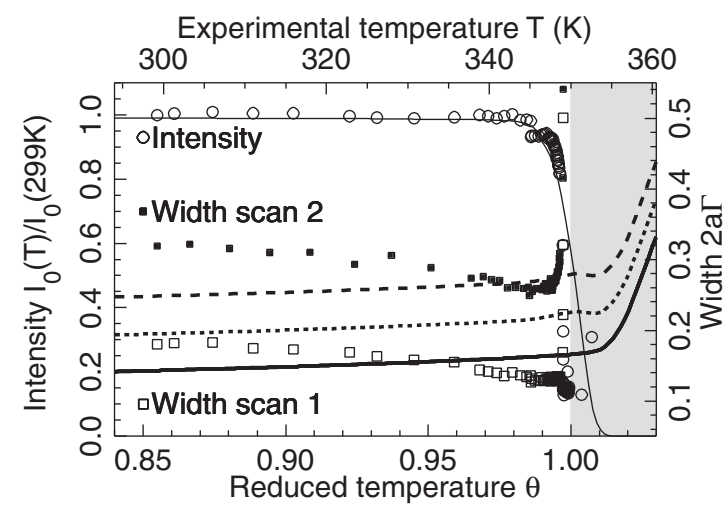

FIG. 11. Comparison between theory and experiment. Points are experimental results, while curves plot theoretical results. A reduced temperature, $\theta=T / T_{c}$, is used, where $T_{c}$ is the temperature at which $50 \%$ of the bases are open. Circles show the integrated intensity of the Bragg peak, rescaled to 1 at low temperature. After rescaling, the results for scans 1 and 2 follow exactly the same curve. The thin line is the calculated integrated intensity. Open squares show the experimental width of scan 1 and the thick solid line is the theoretical value for this width. Filled squares show the width of scan 2 . The dotted line shows the theoretical width of scan 2 calculated by assuming that the longitudinal and transverse structural disorders due to the sequence, determined by conformational analysis [22], are uncorrelated $(\chi=0)$, while the dashed line is the theoretical width of scan 2 calculated by assuming a partial correlation between the longitudinal and the transverse structural disorders $(\chi=0.35)$. The shaded area shows the temperature range in which the experimental observations are hindered by collapse of the sample film. This figure is identical to Fig. 3 in Ref. [16].

The width of the Bragg peak provides the spatial information that standard observations of DNA denaturation cannot give. It is strongly sensitive to the distribution of the sizes of the diffracting clusters. The drop in the average cluster size in the last stage of the transition [Fig. 10(c)] is reflected in the large increase in the width of the Bragg peak predicted by the theory in the high-temperature range [Fig. 10(d)]. For scan 2, with a nonzero transverse component $Q_{\perp}$ of the scattering vector, the width of the peak is also affected by the transverse structural disorder due to the effect of the sequence (variables $\eta_{j}$ ) and by their correlations with the longitudinal structural disorder (variables $\lambda_{j}$ ), measured by the coefficient $\chi$ in Eq. (3). The statistical properties of $\lambda_{j}$ and $\eta_{j}$ have been obtained by conformational analysis [22] but their correlations have not been determined. We show results with $\chi=0$ (no correlation) and $\chi=0.35$ corresponding to moderate correlations. Moreover scan 2 also probes the transverse fluctuations of the bases prior to opening. The inset in Fig. 10(d) shows that, in the vicinity of the transition, these fluctuations are expected to cause an extra increase in the width of the Bragg peak, for scan 2 only. However, this effect is small because the Bragg peak is only generated by the closed sections of the DNA molecules, where the fluctuations are therefore limited to low-amplitude motions of the bases.

\section{DISCUSSION}

To allow a quantitative comparison of the experimental and theoretical results we plot the data as a function of a reduced temperature, $\theta=T / T_{c}$, where $T_{c}$ is the temperature at which $50 \%$ of the bases are open. This is done in Fig. 11. To eliminate the experimental factor associated with the apparatus, in this figure the experimental intensity of the Bragg peak has been rescaled to 1 in the low- $\theta$ limit, which is also the limit of the theoretical intensity in the low-temperature range. The widths have been multiplied by the mean separation of the base pairs $a$, to create dimensionless variables. Therefore a quantitative comparison between theoretical and experimental widths is possible and it does not involve any arbitrary factor.

Let us first examine the integrated intensity of the Bragg peak. Both the experimental data and the theoretical curve show an intensity which stays almost constant up to temperatures very close to $T_{c}, \theta \approx 0.97$. As previously noted this reflects the very low fraction of open base pairs until the vicinity of the transition is reached, in agreement with the theoretical result in Fig. 10(a). In the early stage of the transition the theoretical curve follows the experimental decay of the intensity of the peak, but in the immediate vicinity of $T_{c}(\theta \approx 1)$ the experimental intensity shows an almostdiscontinuous drop, while the theoretical curve has a narrow but smooth decay. This discrepancy is a sign that another phenomenon, not included in the theoretical description, occurs. As discussed in Sec. II, the optical observations of a sample during heating indicate that, at high temperatures, the film itself shows an irreversible "collapse" characterized by a disorganization of the oriented fiber structure. Although this is not described by the model, the theoretical analysis shines some light on this phenomenon. Figure 10(c) shows that, above $T_{c}$, the length of the denatured regions increases very quickly with temperature, almost showing a divergence. As the single strands are very flexible, they can gain a lot of entropy by fully losing their initial orientation so that the remaining double-helix segments are embedded in a liquid-like medium of entangled single strands which quickly becomes the dominant phase in the sample. The rapid growth of the size of the open fragments provides a lot of freedom to the rigid double-helix segments, allowing them to lose their spatial orientation, which causes the sharp drop in the intensity of the Bragg peak. At higher temperatures the sample is no longer a good approximation of a one-dimensional crystal but is more an ensemble of disoriented, short-length DNA This temperature range corresponds to the shaded area in Figs. 10 and 11. It is interesting to note that, when this "collapse" of the film occurs, the theory predicts that the size of the closed segments is still large, of the order of 80-100 base pairs. This is indirectly confirmed by electrophoresis analysis of the length of DNA fragments in the film before and after heating, which indicates that, after film melting, the DNA molecules that were more than 20000 base pairs long at low temperature are chopped into segments of a few hundreds of base pairs. This breaking can be understood by the high stress concentration that occurs at the end of rigid fragments, linked to each other by the flexible single strands, when they rotate as the film melts. It is therefore not surprising to detect DNA 
fragments which have a length of the order of the size of closed clusters.

Let us now examine the width of the Bragg peak. For scan 1 the calculation of the width does not involve any free parameter once the model has been calibrated to match the denaturation curve of DNA. The other parameters entering into the structure factor calculation [Eqs. (5) and (16)] are derived from the structure of DNA [22] and its sound velocity measured along the helix axis [23]. For scan $1\left(Q_{\perp}=0\right)$, the calculation gives a result which is in good agreement with experiments (Fig. 11), although the experimental results are probably affected by some annealing of the sample, causing a slight decay of the width of the peak (Fig. 4) that the theory does not describe. Despite this limitation, two points emerge from the comparison between theory and experiments. First the structural data which enter into the calculation of the width for scan 1, particularly the fluctuations of the base-pair distances along the helix, measured by $\left\langle\lambda^{2}\right\rangle$, corresponding to a standard deviation of $0.18 \AA$, are tested here on a large scale since the width of the diffraction peak involves an average over the billions of base pairs of the sample. The discrepancy of less than $15 \%$ between the calculated and the experimental widths indicates that the results of the conformational analysis [22] are accurate. Second, for scan 1, the width of the peak is remarkably constant until $T_{c}$ and the collapse of the film. This indicates that the base-pair openings, which start to be very significant at $\theta=T / T_{c}=0.98$, do not cause a sharp decrease in the size of the diffracting clusters until denaturation has occurred. This is what the theoretical model indicates [Fig 10(c)]. Clusters of about 100 base pairs remain intact well within the denaturation region and denature as a whole, this being allowed by the surrounding open bubbles in the last stage of the denaturation. This provides a good test of the statistical physics description of DNA that we use, validating the model beyond its ability to predict melting curves.

Contrary to scan 1 , the calculation of the width of the Bragg peak in scan 2, with a transverse component $Q_{\perp}$ of the scattering vector, involves an unknown parameter, coefficient $\chi$, which measures the correlation between the longitudinal and the transverse structural disorder due to the sequence [Eq. (3)]. Figure 11 shows that, if we ignore this correlation by setting $\chi=0$, the theoretical value is about $30 \%$ lower than the experimental width. Setting $\chi=0.35$, that is, a moderate correlation, we get a theoretical width which matches the experimental value for $\theta \approx 0.97$, which we take as the temperature where the sample is "annealed." The results suggest that neutron scattering could be used to probe this structural property of DNA, and it would be interesting to test this result by conformational analysis.

In the range $0.99<\theta<1$ the experiment detects a significant increase in the width of scan 2 which is not shown by the theoretical curve. According to Eq. (5), the transverse fluctuations of base pairs, which become large because they start to open, bring an extra contribution to the width through an increase in $\left\langle\xi^{2}\right\rangle$. This contribution is visible in the inset in Fig. 10, but as discussed in Sec. III, this effect has to be small since only fluctuations in the closed clusters of base pairs can contribute to the shape of the Bragg peak. This is not enough to account for the observed increase in the width of scan 2 below the transition. However, there is another contribution to the width which is not included in the structure factor calculation: the misalignment of the molecules. It is very likely that the collapse of the film is preceded by increased fluctuations in the orientation of the helix fragments. This should have a strong influence on the width of the Bragg peak in scan 2. For instance, orientational fluctuations of $10^{\circ}$, which change the projection of the base pair distance on $Q_{\|}$by less than $2 \%$, lead to a projection of $17 \%$ of this distance along $Q_{\perp}$. Only a theory of the collective effects leading to melting of the film could properly account for this effect.

\section{CONCLUSION}

In conclusion, we have shown that neutron scattering can be used to monitor the thermal denaturation of DNA, providing the spatial information that other methods cannot measure. By focusing the study on the Bragg peak, which is associated with base-pair stacking, we obtain accurate results which are not limited by the fiber nature of the samples. The width of the Bragg peak can be described by a simple nonlinear model for DNA at the scale of base pairs, thus providing further validation of this model, which has already proved to be able to predict complex DNA denaturation curves with a small number of parameters. Moreover, with selection of a scattering vector which is not parallel to the axis of the DNA helix, the shape of the Bragg peak is also sensitive to the transverse fluctuations of base pairs, which this dynamical model can calculate. This should allow further comparison between theory and experiments by investigating not only the opening of base pairs, but also their large-scale fluctuations, important in many biological processes. This aspect could not be investigated in the present experiments due to the collapse of the fiber structure of the sample.

\section{ACKNOWLEDGMENTS}

We want to thank Dr. Monica Jimenez-Ruiz (Institut Laue Langevin) and the IN8 and IN3 instrument teams for helpful assistance. We also thank Emmanuel André for technical help for the optical microscopy observations and Jordi Xiol for assistance with electrophoresis studies. Part of this work has been supported by the program Accueil-Pro program of Région Rhône-Alpes.
[1] M. H. F. Wilkins, A. R. Stokes, and H. R. Wilson, Nature 171, 738 (1953).

[2] R. E. Franklin and R. G. Gosling, Nature 171, 740 (1953).

[3] J. D. Watson and F. H. C. Crick, Nature 171, 737 (1953).
[4] M. Guéron, M. Kochoyan, and J.-L. Leroy, Nature 328, 89 (1987).

[5] R. Thomas, Bioch. Biophys. Acta 14, 231 (1954).

[6] S. A. Rice and P. Doty, J. Am. Chem. Soc. 79, 3937 (1957). 
[7] R. M. Wartell and A. S. Benight, Phys. Rep. 126, 67 (1985).

[8] C. T. Wittwer, Hum. Mutat. 30, 857 (2009).

[9] D. Poland and H. A. Scheraga, J. Chem. Phys. 45, 1456 (1966); 45, 1464 (1966).

[10] M. Peyrard and A. R. Bishop, Phys. Rev. Lett. 62, 2755 (1989); T. Dauxois, M. Peyrard, and A. R. Bishop, Phys. Rev. E 47, R44 (1993).

[11] N. Theodorakopoulos, Phys. Rev. E 82, 021905 (2010); J. Nonlin. Math. Phys. (in press), e-print arXiv:1102.0259.

[12] D. Jost and R. Everaers, J. Phys. Condens. Matter 21, 034108 (2009).

[13] R. A. Cowley, in Methods of Experimental Physics, Vol. 23, part C, edited by K. Sköld and D. L. Price (Academic Press, Orlando, FL, 1987), pp. 1-68.

[14] A. Rupprecht, Acta Chem. Scand. 20, 494 (1966); Biotechnol. Bioeng. 12, 93 (1970).

[15] W. Fuller, T. Forsyth, and A. Mahendrasingam, Philos. Trans. R. Soc. London B 359, 1237 (2004).

[16] A. Wildes, N. Theodorakopoulos, J. Valle-Orero, S. CuestaLópez, J.-L. Garden, and M. Peyrard, Phys. Rev. Lett. 106, 048101 (2011).

[17] H. Grimm, H. Stiller, C. F. Majkrzak, A. Rupprecht, and U. Dahlborg, Phys. Rev. Lett. 59, 1780 (1987).

[18] M. D. Frank-Kamenetskii, Biopolymers 10, 2623 (1971); M. D. Frank-Kamennetskii, Phys. Rep. 288, 14 (1997).

[19] G. W. H. Höhne, W. F. Hemminger, and H.-J. Flammersheim, Differential Scanning Calorimetry (Springer, New York, 2010).
[20] J. Valle-Orero, J.-L. Garden, J. Richard, A. Wildes, and M. Peyrard (unpublished).

[21] X.-Q. Mu, Acta Crystallogr. A 54, 606 (1998).

[22] R. Lavery, M. Moakher, J. H. Maddocks, D. Petkeviciute, and K. Zakrzewska, Nucl. Acids Res. 37, 5917 (2009).

[23] M. Krisch, A. Mermet, H. Grimm, V. T. Forsyth, and A. Rupprecht, Phys. Rev. E 73, 061909 (2006).

[24] M. Peyrard, S. Cuesta López, and D. Angelov, J. Phys. Condens. Matter 21, 034103 (2009).

[25] N. I. Korolev, A. P. Vlasov, and I. A. Kuznetsov, Biopolymers 34, 1275 (1994).

[26] S. M. Lindsay, S. A. Lee, J. W. Powell, T. Weidlich, C. Demarco, G. D. Lewen, J. N. Tao, and A. Rupprecht, Biopolymers 27, 1015 (1988).

[27] T. S. van Erp, S. Cuesta López, and M. Peyrard, Eur. Phys. J. E 20, 421 (2006).

[28] Y.-L. Zhang, W.-M. Zheng, J.-X. Liu, and Y. Z. Chen, Phys. Rev. E 56, 7100 (1997).

[29] N. Theodorakopoulos, Phys. Rev. E 77, 031919 (2008).

[30] Sigma Aldrich, Product information sheet D1626: Deoxyribinucleic acid (DNA) sodium salt from salmon testes.

[31] GenBank [http://www.ncbi.nlm.nih.gov/Genbank/].

[32] G. N. Cohen, V. Barbe, D. Flament, M. Galperin, R. Heilig, O. Lecompte, O. Poch, D. Prieur, J. Quérellou, R. Ripp, J. C. Thierry, J. Van der Oost, J. Weissenbach, Y. Zivanovic, and P. Forterre, Mol. Microbiol. 47, 1495 (2003). 